\title{
The importance of history in definitions of culture: Implications from phylogenetic approaches to the study of social learning in chimpanzees
}

\author{
STEPHEN J. LYCETT \\ University of Kent, Canterbury, England
}

\begin{abstract}
Some have claimed that wild chimpanzees possess multiple socially learned traditions that might constitute cultural patterns. Others, however, have suggested that even fundamental alternative explanations, such as proximate genetic mechanisms, have not been addressed satisfactorily. Multiple analyses using phylogenetic (cladistic) methods, however, have been shown not to support the genetic proposition. Rather, such analyses are more consistent with the growing body of evidence from studies of both wild and captive animals suggesting that behavioral patterns in wild chimpanzees are socially learned. The question remains, however, as to whether, from a scientific viewpoint, it is useful to term such patterns cultural. It is argued here that cultural mosaics of multiple behaviors that differ intercommunally, both in humans and chimpanzees, are an emergent property of a phylogenetic (i.e., historical) process of descent with modification, mediated by mechanisms of social transmission, variation, and sorting through time. This historical perspective is productive when attempting to consider the phenomenon of culture across species.
\end{abstract}

\section{The Controversy of Culture}

The question of whether culture exists in other animals (Galef, 1992) was never likely to be settled easily. A scientific response to the issue poses many difficult questions: How do we define culture? How do we recognize it in other animals? How do we exclude alternative explanations for our observations? For some, the controversial nature of the debate is as close to intellectual warfare as any scientific argument might come (Kendal, 2008; McGrew, 2003, 2009). Yet, despite the challenging nature of this issue, it is one of fundamental importance. It is one that comes close to (re)negotiating our conceptual place in the biological world and how we regard other species (Corbey, 2005).

An argument that has difficulty defining what it may even be debating is always set for trouble, and defining culture to the satisfaction of all has proved to be an immense stumbling block (McGrew, 2004). Traditionally, for some workers (especially anthropologists), an easy resolution to such a question was to simply define culture as that which humans possess that other animals do not (see, e.g., Tylor, 1871). As Laland and Hoppitt (2003) noted, however, such an exclusive definition is problematic, because it immediately inhibits any chance of an evolutionary approach to an understanding of human culture (i.e., looking for the roots of the phenomenon). Recently, however, something of a consensus has emerged on defining at least one central feature of culture. That is, for a behavior to be seen as potentially cultural, it must be shared by several individuals and learned via a process of social interaction (e.g., Laland, 2008; McGrew, 2004). The corollary of this, as the accompanying articles in this issue testify, is that there has been an increased research focus on discerning whether particular animals might learn behaviors socially.

Claims that animals learn and share behaviors in the wild through social learning are now widespread. Such claims exist, for example, in fish (Brown \& Laland, 2003), cetaceans (Krützen et al., 2005; Rendell \& Whitehead, 2001; Sargeant \& Mann, 2009), birds (Madden, 2008), and rats (Terkel, 1996). Arguably, however, the most well-known claims of social learning in animals are those pertaining to primates, and in particular, the great apes. Indeed, a landmark event in the study of animal behavior came over 10 years ago with the publication of recorded intercommunity behavioral differences among seven long-term chimpanzee (P. troglodytes) study sites (Whiten et al., 1999). Over 30 years' worth of observations in the wild had begun to foster the notion that diverse sets of socially learned traditions were present in chimpanzee communities (Boesch, 1996; Boesch \& Boesch-Achermann, 2000; Goodall, 1986; McGrew, 1992; Nishida, 1990; Wrangham, McGrew, de Waal, \& Heltne, 1994). Whiten et al.'s (1999) systematic collation of this information, involving the site directors of the most intensively studied communities, appeared to corroborate the potential existence of community-specific profiles involving multiple behaviors ( $n \geq 39$ putative socially learned traditions). Using 
the same approach, van Schaik et al. (2003) subsequently made comparable claims for a behavioral diversity in orangutans (Pongo) ( $n \geq 19$ putative traditions), while Hohmann and Fruth (2003) made the case for bonobo chimpanzees (P. paniscus), the sister taxon of P. troglodytes ( $n \geq 14$ putative traditions).

As Whiten and van Schaik (2007) noted, the claim that such behavioral patterns are socially learned in the wild is based partly on instances of novices (usually juveniles) being observed paying close attention to adult instances of the behavior. Support for this line of reasoning comes from field experiments such as those undertaken with the chimpanzee (P. $t$. verus) community at Bossou (Guinea; Biro et al., 2003; Biro, Sousa, \& Matsuzawa, 2006; Matsuzawa et al., 2001). Here, two novel species of nut (Coula and Panda) cracked by neighboring communities were deliberately introduced to the Bossou group, who normally only cracked the locally available oil-palm nuts (Elaeis). When Coula nuts were first introduced to the group in 1993 (Matsuzawa, 1994), the reaction of several individuals was to nibble or sniff the novel items, while other individuals simply ignored them. A single adult female, however, immediately cracked the nuts without hesitation and did not pause to investigate the novel objects. As was argued by Biro et al. (2003; Biro et al., 2006), this is consistent with the suggestion that this adult female had migrated from a neighboring group and had prior experience with this species of nut. Her nut-cracking behavior subsequently attracted attention from juveniles, and by the fourth session of Coula presentation, two of these juveniles had also begun to crack the nuts (Matsuzawa et al., 2001). By 2002, as much as $67 \%$ of the population at Bossou were cracking Coula nuts (Biro et al., 2003; Biro et al., 2006). Panda nuts (introduced in 2000) did not attract the same attention, and although these were cracked successfully by two adult females, they did not persist in this behavior (Biro et al., 2003). Two juveniles briefly tried to crack Panda nuts, but were unsuccessful. It seems that in the two contrasting fates of these nutcracking behaviors, sustained exhibition was essential for the successful transmission of Coula nut cracking to the group over subsequent years. Equally suggestive is a longitudinal study of termite-fishing patterns at Gombe (Tanzania) by Lonsdorf, Eberly, and Pusey (2004). At this study site, it was demonstrated that female chimpanzees gain proficiency in termite-fishing quicker than males and with greater fidelity of technique when compared with their mother. This pattern is consistent with observational social learning, since Lonsdorf et al. also found that young females spend a significantly greater amount of time observing their mothers fish for termites than do young males.

Recent experimental work with captive groups has provided further evidence that chimpanzees possess at least the capability to propagate and sustain social traditions of the type purported to be operational in the wild. In a range of experimental studies, the diffusion method has been utilized, whereby a novel behavior is seeded within a community and its spread across the community is documented (e.g., Bonnie, Horner, Whiten, \& de Waal, 2007;
Hopper et al., 2007; Horner, Whiten, Flynn, \& de Waal, 2006; Whiten, Horner, \& de Waal, 2005; Whiten et al., 2007). Such studies typically involve chimpanzees performing a particular tool-use technique in order to gain a food reward. Initially, these involved two individuals (high-ranking females) from two separate groups being trained privately in alternative tool techniques to obtain the same result (i.e., remove the food reward from the experimental apparatus). Thereafter, the trained individuals were reintroduced to their respective groups along with the experimental apparatus in order to determine whether the particular technique would spread in the form of a tradition. These experiments were successful in demonstrating that the predominant technique used in each group was that performed by the trained individuals (Whiten et al., 2005). Other studies have meanwhile demonstrated individual instances of transmission from one chimpanzee to another over multiple generations, demonstrating high fidelity in transmission chains of up to six individuals (Horner et al., 2006). Subsequent experiments have also demonstrated that when a group is practicing a seeded tool technique, they may transmit that technique to further groups (Whiten et al., 2007). Collectively, these studies form some of the most compelling evidence yet provided that chimpanzees possess the necessary components required to learn and sustain the multiple tradition patterns claimed to exist in the wild.

Despite the documentation of behavioral diversity and the highly suggestive nature of observations of chimpanzees both in the wild and in captivity, several objections to the notion that wild chimpanzees genuinely possess culture have been offered persistently over the years and continue to be so (see, e.g., specific chapters in Laland $\&$ Galef, 2009). One such objection centers on whether the behavioral diversity seen in chimpanzees is actually socially learned and might better be explained by alternative factors such as genetic mechanisms or environmental influences. Another category of argument harks back to older concerns regarding whether the precise nature of chimpanzee traditions - even if they are socially learned - is fundamentally different from that of human culture in specific characteristics (e.g., they do not involve direct teaching or specific cognitive mechanisms such as true imitation) such that they do not genuinely merit being termed cultural. Such objections are reasonable, at least to the extent that they require a scientifically grounded response. Given that chimpanzees are our closest living relative, the focus on whether chimpanzees might qualify for culture in the context of both categories of debate is especially important, since if chimpanzees can be excluded as cultural animals, the recognition of a genuinely phylogenetically homologous cultural capacity (with that of humanity) - at least in extant animals - escapes us.

The objection concerning the potential role of genetics in regard to the issue of chimpanzee culture is based partly on methodological concerns (Laland \& Janik, 2006). Whiten et al.'s (1999) claim that the behavioral diversity exhibited by chimpanzees is cultural was based on a procedure that has subsequently come to be termed the method of exclusion. When this method is applied, 
instances of behavioral diversity among communities are tabulated (i.e., presence, absence, frequency of observation) and subsequently compared, with a view to identifying potential environmental causes of the behavioral variation. Thereafter, the conservative step is taken of excluding behaviors for which all recorded instances of absence are explicable by environmental dictates. In essence, a behavior is deemed socially learned via this method if it is observed frequently in at least one community, exhibits cross-community variation in terms of presence, and survives the environmental screening stage. Hence, although this method pays some attention to environmental influences on behavioral diversity, it does not directly address the potential of proximate genetic factors (Galef, 2009; Laland \& Hoppitt, 2003; Laland \& Janik, 2006; Laland, Kendal, \& Kendal, 2009). As Laland and Janik (2006) noted, this is particularly pertinent in the case of chimpanzees, since as many as one third of the behavioral variants documented by Whiten et al. (1999) are observed in a single subspecies, the existence of which is supported by genetic studies demonstrating genetic isolation for hundreds of thousands of years (e.g., Gagneux, Gonder, Goldberg, \& Morin, 2001; Gonder, Disotell, \& Oates, 2006).

For those workers that accept (at least in principle) the case for social learning in wild chimpanzees, arguments against use of the term culture to describe the behavioral patterns that emerge from such mechanisms take a variety of forms. Each, however, focuses on specific definitional issues with regard to the term culture. For instance, Tomasello (1999) argued that a key feature of human culture as opposed to chimpanzee traditions is the accumulation of modifications over time, or what he termed the ratchet effect. Recent observations suggest, however, that to assert a total absence of ratcheting in some instances of wild chimpanzee behavior may be an oversimplification. For example, recent studies in the Goualougo Triangle (Republic of Congo) have shown that chimpanzees (P. t. troglodytes) in this area modify termite-fishing probes to produce a brush-tipped version of the standard fishing probe used by some other communities (Sanz, Call, \& Morgan, 2009). Experimental work additionally demonstrated that brushtipped probes are more effective in gathering termites than unmodified versions, suggesting that the deliberate actions taken to modify the brush-tipped version are an elaboration on the basic design (Sanz et al., 2009). Use of tool sets in sequential stages during bouts of termite fishing (e.g., Sanz, Morgan, \& Gulick, 2004) or honey extraction (e.g., Boesch, Head, \& Robbins, 2009) also indicate the potential for accumulated elements in certain chimpanzee behaviors. These examples argue against imposition of a strict presence/absence ratchet effect dichotomy in the case of humans versus chimpanzees, despite the comparatively impressive extent of ratcheting in many human behaviors and traditions. As McGrew (2009) pointed out, however, a further potential problem with the ratchet effect argument against use of the term culture for chimpanzee traditions is that many instances of human cultural behavior exhibit considerable stasis, rather than change through time. Of course, if socially learned traditions are adaptive, stasis- rather than consistent mutation - may be a fundamental component of such behaviors (Palmer, 2010).

Other arguments against application of the term culture to chimpanzees focus on specific social learning mechanisms, such as imitation or teaching, or define human culture by specific properties such as symbol use. Imitation, whereby an individual focuses on both the goal of a task and reproducing the actions of others in achieving that goal, was argued to be absent in chimpanzees (Tomasello, 1994). In other words, chimpanzees may emulate the goals of others, but they do not imitate in strict terms and so a distinct element of human cultural transmission (so the argument went) is absent in the case of chimpanzees. Tomasello (2009) has subsequently revised this view in light of experimental work demonstrating that chimpanzees appear to distinguish between intentional and nonintentional actions when copying the means of achieving a goal (Call \& Tomasello, 1998). It has also been shown that arbitrary conventions introduced in experimental settings may diffuse within a community, which is indicative of imitative rather than emulative learning (Bonnie et al., 2007). It is true, however, that claims for intentional teaching in chimpanzees remain rare (but see Boesch, 1991). Given the diversity of means by which humans learn social conventions and traditions without deliberate pedagogy, however, the question remains as to whether it is useful to establish teaching as the definitive element of human culture (see also Thornton \& Raihani, 2010, and Hoppitt et al., 2008, for further discussion of the definition of teaching and its occurrence in nonhuman animals). Applying such a definition strictly could potentially lead to the exclusion of a wide section of human cultural diversity (McGrew, 2004). Teaching, therefore, may be an important part of some human cultural institutions (Tehrani \& Riede, 2008) but may not necessarily be a definitive marker. A similar problem besets the use of symbolism (e.g., Tuttle, 2001) as a diagnostic, to say nothing of the debate concerning a potential capacity for symbol use in chimpanzees (Brumann, 2002; McGrew, 2004). In summary, these definitional points perhaps provide some of the most controversial arguments against applying the term culture to chimpanzees, aggravated by the fact that they frequently seem resistant to a strict present-in-humans/absent-in-chimpanzees dichotomy and/or that they are difficult to operationalize consistently in terms of what is appropriately termed cultural in the human versus chimpanzee case.

Systematic documentation of behavioral diversity in wild animals (e.g., van Schaik et al., 2003; Whiten et al., 1999; Whiten et al., 2001) has influenced a whole new era in the study of animal social learning, both in the wild and in captivity. However, the foregoing discussion also illustrates that such studies have not necessarily led to a quieting of the controversy surrounding culture. If anything, they have been responsible for turning up the volume on this already vociferous debate (see, e.g., recent exchanges between Laland \& Janik, 2007, and Krützen, van Schaik, \& Whiten, 2007, and chapters in Laland \& Galef, 2009). Ironically, even primatologists themselves have become involved in debates concerning how culture might be properly (re)defined so that it might be distinguished from 
social learning and tradition per se. Thus, adding further controversy to this mix are claims by some proponents of ape culture that social learning alone is insufficient to identify culture: Animals must exhibit multiple socially learned behavioral patterns to be genuinely cultural, it is claimed (e.g., McGrew, 2004; Whiten \& van Schaik, 2007). Such a definition shifts emphasis from merely the proximate means by which behavioral patterns are propagated (i.e., social learning) to a quantitative parameter. As such, animals that exhibit just one - even unambiguoussocially learned tradition in the wild would be excluded as cultural creatures.

\section{Applying Cladistic Methods to the Chimpanzee Culture Controversy}

The question of whether putative cultural variations in animal behavior might be genetically determined rather than the result of social learning mechanisms remains pertinent (Galef, 2009; Laland et al., 2009). Given the complexity of some of the behaviors involved in this debate in the case of chimpanzees (e.g., termite fishing), it may seem intuitively implausible that genetic differences could account for the intercommunity differences of P. troglodytes (see, e.g., de Waal, 1999). However, as Whiten and van Schaik (2007) recently noted, studies of non-sociallylearned tool manufacture and use by hand-reared woodpecker finches (Tebbich, Taborsky, Fessl, \& Blomqvist, 2001) and New Caledonian crows (Kenward, Weir, Rutz, \& Kacelnik, 2005) demonstrate the potential for genes to underlie behaviors that intuitively seem unlikely to be genetically influenced (see also Thouless, Fanshawe, \& Bertram, 1989). As such, the genetic explanation for the population-level variation in chimpanzee behavior cannot be simply dismissed out of hand, especially, as Laland and Janik (2006) pointed out, given the support from genetic studies indicating subspecies-level distinctions within Pan troglodytes. This has profound implications: In effect, if the behavioral differences between chimpanzee communities are the product of genetic mechanisms rather than social learning mechanisms, all debates regarding issues such as ratcheting in chimpanzees and the importance (or otherwise) of multiple behaviors in properly defining $\mathrm{cul}$ ture become irrelevant.

It is this central question of whether genetics might provide a predominant explanation for chimpanzee behavioral diversity that colleagues and I have attempted to tackle in a series of studies (Lycett, Collard, \& McGrew, $2007,2009,2010)$. In order to achieve this, we have utilized a phylogenetic method (i.e., cladistics) more typically applied to genetic and phenotypic data in order to determine evolutionary relationships in living and fossil taxa (e.g., Argue, Morwood, Sutikna, Jatmiko, \& Saptomo, 2009; Gilbert \& Rossie, 2007; Liu, Rubidge, \& Li, 2010; Sperling et al., 2009; Todd, 2010). Cladistic analysis is based on the parsimonious null model of taxonomic bifurcation and character evolution. A succinct definition of cladistic analysis has been provided by Brower (2000, p. 13), who stated that it is a "method of grouping by parsimonious patterns of shared character state change." As is widely known, cladistics emphasizes the importance of using traits that are uniquely shared (i.e., shared-derived) by two or more taxa, rather than shared primitive characteristics (symplesiomorphies), unique features possessed by a single taxon, or convergences (homoplasies) in determining the phylogenetic relationships between evolved units.

Cladistics is notorious for its association with esoteric terminology, which is particularly unfortunate when wishing to apply the method outside of its more typical arena of usage (see Table 1 for definitions of key terms). However, in recent years, several accessible introductions to the principles and terminology of cladistics have become available (e.g., Kitching, Forey, Humphries, \& Williams, 1998; McLennan \& Brooks, 2001), including some written specifically for disciplines outside the more typical sphere of application (e.g., O'Brien \& Lyman, 2003). It has also been noted that despite the use of rather complex computer algorithms to determine the most parsimonious cladograms, cladistics can be conceptually broken down into a small series of fundamental methodological steps (McLennan \& Brooks, 2001). The first step in any cladistic analysis is to delineate the taxonomic units (i.e., to identify those units that one wishes to understand the structure of relationships between). In phylogenetic terminology, these analytical units are commonly referred to as operational taxonomic units (OTUs). The second stage is to generate a character state matrix describing the character states for each OTU. Next, the direction of evolutionary change (character polarity in cladistic terminology) is determined, most commonly via comparison with an outgroup. An outgroup is a taxon known to be closely related to the other taxa in the analysis (the ingroup), but one that can confidently be assumed to have diverged from those taxa at an earlier phylogenetic point than the ingroup taxa diverged from each other (Table 1). Thereafter, a branching diagram or tree (i.e., cladogram) is constructed that describes the relationships between OTUs for each character. Finally, in accordance with the principle of parsimony, an ensemble cladogram is constructed that is consistent with the largest number of character trees and also, therefore, requires the least number of ad hoc (nonparsimonious) character state changes to be invoked in order to explain the phylogenetic relationship between the different OTUs. This use of parsimony also explains why cladograms are frequently referred to as maximum parsimony (MP) trees.

The initial studies designed to test the genetic hypothesis utilized Whiten et al.'s (1999) major cross-community data set of 39 putative cultural variants (Lycett et al., 2007, 2009). Working through the procedure just described, the construction of an initial cladogram based on this data involved the following stages: First, following Whiten et al. (1999), taxonomic units were taken to be the seven chimpanzee (P. troglodytes) communities subject to the most long-term scientific observation. Specifically, the OTUs in this instance were, therefore, the communities at Bossou (Guinea), Taï Forest (Côte d'Ivoire), Gombe (Tanzania), Mahale K and Mahale M groups (Tanzania), Kibale Forest-Kanyawara (Uganda), and Budongo Forest (Uganda). Following the coding procedure of Whiten 
Table 1

Key Cladistic Terms and Definitions

Cladistic Term

Operational Taxonomic Units (OTUs)

Character

Character state

Outgroup

Clade

Node

Synapomorphies

Plesiomorphies

Symplesiomorphies

Autapomorphic

Homologous

Homoplasy (pl. homoplasies)

Tree length

Monophyletic

\section{Definition}

The basic taxonomic units used in the analysis (i.e., the units for which an understanding of relationship is required).

Characters are attributes that describe specific properties or features of the OTUs. These are sometimes binary in form (e.g., a particular feature is present or absent) but may also involve multiple character states (e.g., absent, present, habitual, customary).

A character state is the particular form of character possessed by an individual OTU (e.g., absent, present, habitual, customary).

An outgroup is a taxonomic unit that is closely related to the main OTUs used in the analysis. The outgroup is used for comparative purposes to determine the direction of evolutionary (character state) changes in a tree.

A group of taxonomic units comprising their common ancestor and all of its descendents. Such groups are said to be monophyletic.

Nodes are the junctions (joints) of branches in a tree diagram (or cladogram) drawn to represent the hypothesized evolutionary relationships of taxonomic units.

Synapomorphies are character states that are uniquely shared between two or more closely related species comprising a clade. The presence of synapomorphies is particularly important in determining which taxonomic units are most closely related (i.e., deciding which units should be linked together to form a clade).

Plesiomorphies are the particular character state(s) that are seen in the taxonomic units that form the lowest branches of a cladogram (tree) closer to the outgroup. As such, they are sometimes referred to as primitive character states, but it should be emphasized that no specific judgmental connotations in terms of quality or value necessarily accompany this term.

Symplesiomorphies are primitive character states (see plesiomorphies) shared by two or more taxonomic units.

An autapomorphic feature is a character state uniquely possessed by only one taxonomic unit. In being unique in this sense, autapomorphic features cannot be used to determine evolutionary relationships.

A homologous feature refers to a character state shared between two or more taxonomic units as a result of descent from a common ancestor (compare with homoplasy).

Homoplasy is the presence of the same character state in two or more taxa that are not closely related. Instances of homoplasy can occur by processes of convergent or parallel evolution or by character state reversal (i.e., when a taxonomic unit reverts back to a plesiomorphic character state exhibited by taxa to which it is not most closely related).

The number of evolutionary changes (or steps) required by the tree to explain the distribution of character states among the taxa.

A monophyletic group (or clade) is a group of taxa closely related by means of descent from a common ancestor. et al. (1999), character states for each of the 39 putative cultural variants were coded in the matrix as $0=$ absent, 1 = present, 2 = habitual, 3 = customary, ? = status uncertain. Character states were treated as ordered and freely reversing in the phylogenetic analysis (i.e., a change in state, for example, from 1 to 2 is equivalent in cost to that of 2 to 1 ), thus ensuring that directionality of character state change was not determined a priori. Character polarity was subsequently determined via application of the outgroup method. In this instance, behavioral data for Pan paniscus (Hohmann \& Fruth, 2003) were used as the outgroup, since this taxon is the closest living relative of $P$. troglodytes. Thereafter, all data were imported into the commonly used phylogenetic reconstruction program PAUP*4.0 (Swofford, 1998), and the most parsimonious cladogram was identified using this program's "branch and bound" search algorithm, which is guaranteed to find the most parsimonious tree. The MP tree produced via this procedure when utilizing all seven $P$. troglodytes communities is shown in Figure 1.

Constructing a phylogenetic tree on the basis of the Whiten et al. (1999) data set was, however, only the initial stage in testing the genetic hypothesis for chimpanzee behavioral patterning. Testing the predictions of this hypothesis depended on a number of subsequent steps involving tree statistics that describe phylogenetic structure within the tree and permutation tests, which can also be used to determine the levels of phylogenetic structure. The predictions derived from the genetic hypothesis were determined from our current state of knowledge regarding chimpanzee genetic patterning. In the case of the chimpanzee communities included in the Whiten et al. (1999) data set, five communities are generally described as belonging to the East African subspecies P. t. schweinfurthii (i.e., Gombe, Kibale Forest-Kanyawara, Budongo Forest, Mahale $\mathrm{K}$ and Mahale $\mathrm{M}$ groups), whereas the other two communities (i.e., Bossou and Taï Forest) are typically described as belonging to the subspecies $P$. $t$. verus. Analyses of genetic data have confirmed that East African P. t. schweinfurthii and West African verus are well differentiated from each other phylogenetically, whereas communities within East Africa do not exhibit equivalent phylogenetic differentiation (Gagneux et al., 2001; Gagneux et al., 1999; Gonder et al., 2006). Such partitioning in the genetic data suggests a straightforward prediction if the primary underlying cause of the behavioral patterning is genetic at the proximate level. That is, there should be stronger between-subspecies phylogenetic structure to the 


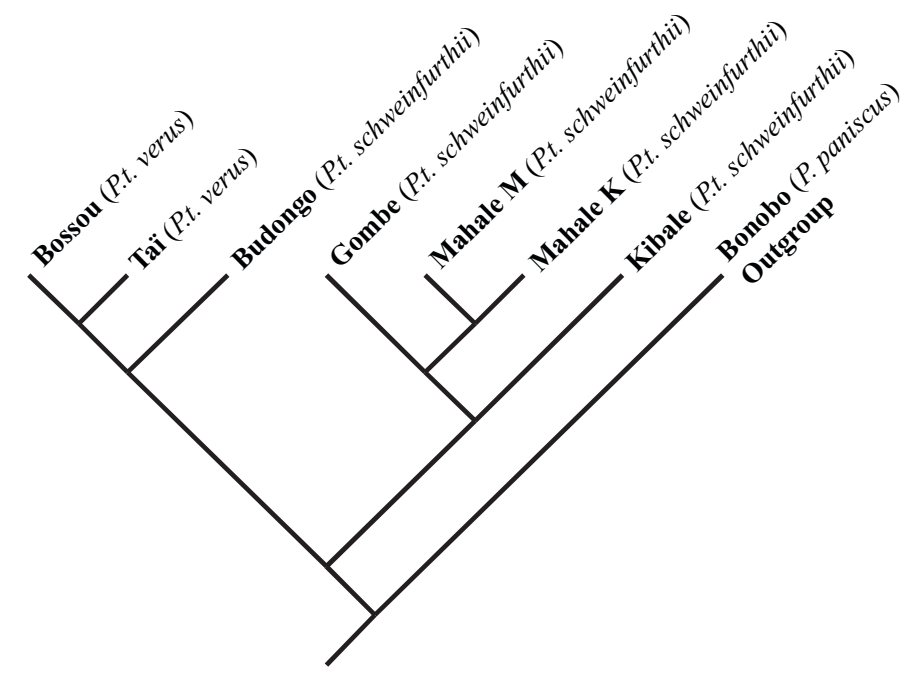

Figure 1. Maximum parsimony tree produced by cladistic analysis of 39 putatively cultural chimpanzee behavioral variants (tree length $=$ 177 , RI = .44). From "Phylogenetic Analyses of Behavior Support Existence of Culture Among Wild Chimpanzees," by Lycett, Collard, and McGrew, 2007, Proceedings of the National Academy of Sciences, 104, pp. 17588-17592. Copyright 2007 by the National Academy of Sciences. Adapted with permission.

behavioral data than there is within subspecies. In other words, the behavioral data should match the genetic data in terms of structure.

Lycett et al.'s (2007) initial test of this prediction involved the use of a widely used tree statistic known as the retention index (RI). This statistic measures the number of homoplastic changes in a cladogram independent of its length (i.e., the number of evolutionary changes; Farris, 1989a, 1989b). Homoplastic changes may be the product of convergent/parallel evolution or processes such as character state reversal. Essentially, the RI is a measure of goodness of fit to a bifurcating tree model and, in turn, describes how well similarities and differences across a group of taxa can be explained by a given phylogenetic hypothesis. A maximum RI of 1 indicates that the cladogram requires no homoplastic change to fit the phylogenetic hypothesis in question, whereby the level of homoplasy increases as the index approaches 0 . The $\mathrm{RI}$ is a useful goodness-of-fit measure when comparing across different data sets, because unlike some alternative measures (e.g., the consistency index), it is not affected by the number of taxa or the number of characters (Kitching et al., 1998).

As noted earlier, if the genetic hypothesis is to be supported, there should be stronger between-subspecies phylogenetic structure to the behavioral data than there is within subspecies. Accordingly, taking all of these factors into account, Lycett et al. (2007) reasoned that if the predominant cause of the chimpanzee behavioral patterning is genetic, the RI value of the MP tree that includes all seven P. troglodytes communities should be higher than the RI of an MP tree that only includes the five East African communities belonging exclusively to $P$. $t$. schweinfurthii. The results of this analysis were inconsistent with the genetic hypothesis, producing an RI value of .44 for the intersubspecies analysis, while producing an RI value of .53 in the intrasubspecies analysis. Note that it is not necessary to determine whether these RI values are statistically different in order to be in contradiction with the genetic hypothesis. This is because the genetic hypothesis predicts a marked decrease in phylogenetic structure when using the reduced data set as opposed to the all-regions data set. Thus, even an equal RI value (i.e., nonsignificantly different) in both parsimony analyses would be inconsistent with the predictions of the genetic hypothesis. Hence, a statistical difference between the RI values is not essential to reject the genetic hypothesis in the face of an increased RI value when moving from the extended data set to the reduced data sets used in these analyses. On the basis of these results, Lycett et al. (2007) thus concluded that a predominantly genetic causation for chimpanzee behavioral patterns was not supported by the data.

Subsequently, Lycett et al. (2009) considered how the RI values from these analyses compared with those of other data sets taken from the existing literature. In order to do this, they examined the RI values of 25 human cultural data sets and 25 biological data sets (see Tables 1 and 2 in Lycett et al., 2009). The collective RI values of these contrasting sources of data were not statistically different from each other (Mann-Whitney $U=308, p=.930$ ). Given that the biological data sets can confidently be assumed to have been structured by the branching process of speciation, Lycett et al. (2009) concluded that the human cultural data sets were also most likely, on average, to have been structured by an equivalent degree of diversification and branching, rather than intergroup transmission. The RIs for the 25 human cultural data sets had a mean of 60 with a range of .42-.80, whereas the RIs for the biological 
data sets had a mean of .61 and ranged from .35-.94. As was noted above, the cladogram of chimpanzee behaviors determined by Lycett et al. (2007) returned an RI of .44, whereas the regional (P. t. schweinfurthii only) cladogram had an RI of .53. Thus, although these RI values fall toward the lower end of the range, the RI of both chimpanzee cladograms falls within the range of RI values yielded by the human cultural data sets, and the RI value of the single subspecies falls close to the mean of the human cultural RIs. Broadly, this suggests that the cladograms of the chimpanzee behaviors are as tree-like as the human cultural data sets.

Despite these results, one potential shortcoming of the Lycett et al. (2007) analysis is that only one measure of phylogenetic structure was utilized. In addition to the RI, two other commonly used measures of phylogenetic structure are the phylogenetic bootstrap and the permutation tail probability (PTP) test, and it is prudent to determine whether these various methods corroborate each other. Hence, in order to address this, Lycett et al. (2009) repeated their initial analysis using these additional quantitative measures of phylogenetic structure. The phylogenetic bootstrap is routinely used to determine the support for given clades in phylogenetic studies (e.g., Argue et al., 2009; H. F. Smith \& Grine, 2008). The procedure involves a random resampling of characters with replacement and the formation of a large number (typically $\geq 1,000$ ) of pseudoreplicate character matrices with the same number of characters and character states as the raw matrix. Subsequently, MP trees are produced for the pseudoreplicate matrices, and the results are most commonly displayed as a consensus tree with the percentage of pseudoreplicate matrices supporting the original pattern displayed alongside the base (or node) of each clade. Lycett et al. (2009) undertook this procedure using 10,000 pseudoreplicate character matrices. Using the same logic as that of their previous analysis, they reasoned that if the behavioral data are consistent with the genetic hypothesis, the phylogenetic structure should be higher when all seven communities from both $P$. t. verus and P. t. schweinfurthii are included, as opposed to when the East African data set is analyzed alone. However, this procedure returned a higher average bootstrap value for the intrasubspecies analysis than for the intersubspecies analysis (Figure 2). Hence, these new results were consistent with the Lycett et al. (2007) study in contradicting a key prediction of the genetic hypothesis.

The PTP test, additionally used in Lycett et al.'s (2009) follow-up study, also involves randomization of the original character matrix. The PTP test was originally suggested to be a statistical test of phylogenetic signal (Archie, 1989; Faith \& Cranston, 1991). However, subsequent criticism has led to the opinion that the test is better considered a heuristic device for determining relative phylogenetic signal, rather than a statistical test (Kitching et al., 1998). It is in this comparative, heuristic capacity that the test was used by Lycett et al. (2009). The procedure involves permutating the original matrix a large number of times without replacement, creating a predefined number of pseudoreplicate matrices. The lengths (i.e., the number of evolutionary changes required by the tree to explain the distribution of character states among the taxa) of MP cladograms recorded from each of these replicate matrices are then compared with the length of the MP tree constructed from the unpermuted matrix. The higher the number of permuted trees (expressed as a percentage of the total) that are shown to have a longer length than that of the MP tree on the basis of the unpermuted matrix, the stronger the degree of phylogenetic signal (i.e., phylogenetic structure) in the raw matrix.

Using the same reasoning as before, Lycett et al. (2009) tested the structural prediction of the genetic hypothesis utilizing 10,000 permutated matrices. Their results determined that $95.13 \%$ of the 10,000 permuted matrices based on the data set including both subspecies were longer than the MP tree based on the raw matrix, whereas $98.44 \%$
A

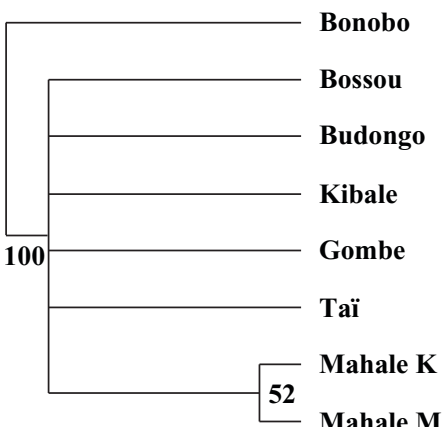

B

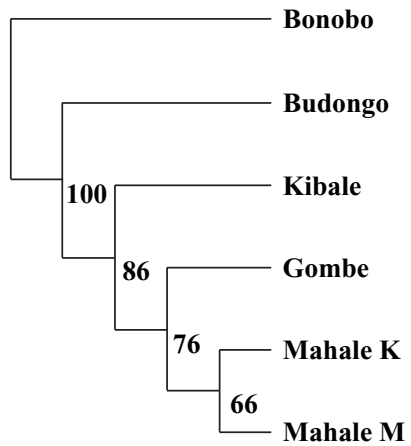

Figure 2. (A) Consensus cladogram of 10,000 bootstrapped trees using both the five East African (P. t. schweinfurthii) and the two West African (P. t. verus) chimpanzee study groups. (B) Consensus cladogram of 10,000 bootstrapped trees using just the five East African study groups. From "Cladistic Analyses of Behavioural Variation in Wild Pan troglodytes: Exploring the Chimpanzee Culture Hypothesis," by Lycett, Collard, and McGrew, 2009, Journal of Human Evolution, 57, pp. 337-349. Copyright 2009 by Elsevier. Adapted with permission. 
were longer in the case of the East African matrix. Hence, in a manner inconsistent with the genetic predictions, phylogenetic structure was deemed higher when only the East African communities were analyzed than when both West African and East African communities were analyzed. Again, since the genetic hypothesis predicts a marked decrease in phylogenetic structure when using the reduced data set (rather than the, albeit slight, increase actually observed), it is not necessary to determine whether the values produced - both in the phylogenetic bootstrap analysis and in the permutation test - are statistically different in order to robustly contradict the genetic hypothesis.

In summary, the three different measures of phylogenetic structure utilized by Lycett et al. $(2007,2009)$ were consistently in conflict with the main structural prediction of the genetic hypothesis. In combination, therefore, there appears to be strong evidence to reject the notion that genetic differences are the predominant, proximate cause of the intercommunity behavioral differences observed in wild chimpanzees. However, if the history and hostility of the chimpanzee culture debate tells us anything, it is that the genetic hypothesis will not fall quite that easily. Indeed, commenting on the issue of animal culture recently, Galef (2009) highlighted a quote from White (2006, p. 472), stating, "All scientific hypotheses should be poked and prodded, tested and retested, and made to stand up to the available observations." It is doubtful that anyone professionally engaged in the animal cultures debates would disagree.

With such considerations in mind, Lycett et al. (2010) again recently put the genetic hypothesis under scrutiny via the application of phylogenetic methods to different data. This new set of analyses was designed to address a potential shortcoming in the behavioral data set used in their previous studies. The major multisite behavioral data set of Whiten et al. (1999) suffers from a limitation of geographic coverage, most notably with regard to a lack of populations from central Africa. Such geographic bias in the Whiten et al. (1999) data set is understandable, given the history of chimpanzee research in Africa, with the earliest fully habituated chimpanzee communities occurring in western and eastern regions. Conversely, chimpanzee communities in central regions of Africa (P. t. troglodytes) have not been subject to the same degree of long-term human observation as those in East and West Africa. In order to overcome this methodological shortcoming, Lycett et al.'s (2010) recent analysis thus focused on tooluse data. Although this reduced the number of behaviors studied, it had the advantage of ensuring that two communities from central Africa could be included, since even when chimpanzee behaviors cannot be observed directly, artefactual and other physical evidence of tool use is still amenable to study (e.g., Koops, McGrew, \& Matsuzawa, 2010; McGrew, 1992; Sanz \& Morgan, 2007; Tutin, Ham, \& Wrogemann, 1995).

In order to carry out these new analyses, data for a total of 10 Pan troglodytes communities were taken from Whiten et al. (1999, 2001) and Sanz and Morgan (2007), with data for Pan paniscus taken from Hohmann and Fruth (2003) for the purposes of outgroup comparison.
The P. troglodytes data were thus composed of communities from Bossou (Guinea), Taï Forest (Côte d'Ivoire), Assirik (Senegal), Gombe (Tanzania), Mahale K group (Tanzania), Mahale M group (Tanzania), the Kibale Forest-Kanyawara community (Uganda), Budongo Forest (Uganda), as well as Goualougo (Republic of Congo) and Lopé (Gabon) from central regions. The tool-use data comprised a subset of 19 categories of tool use, which were considered by Whiten et al. (1999) to represent putative cultural variants, since environmental factors did not appear to explain all occurrences of absence. Such tool-use behaviors include actions such as leaf clipping, whereby an individual noisily rips a leaf in order to gain the attention of others, as well as activities such as the use of wood or stone hammers to crack open nuts. Details and definitions of all tool-use behaviors used in the analysis may be found in Lycett et al. (2010, Tables 1 and 2). Following Sanz and Morgan, character states for each category of tool use were coded as $0=$ absent, $1=$ rare, $2=$ regular, and ? = status uncertain.

As in our previous analyses, we reasoned that if the genetic hypothesis for chimpanzee behavioral patterning is to be supported, phylogenetic analyses of behavior should match the genetic data in terms of structure. Phylogenetic analyses of mitochondrial DNA provide evidence that western chimpanzees are well differentiated from both central and eastern chimpanzee communities genetically (Gagneux et al., 2001; Gagneux et al., 1999; Gonder et al., 2006). However, these analyses also suggest that central and eastern communities are not reciprocally monophyletic (i.e., they do not form two distinct clades, each with a separate, unique common ancestor). Hence, the genetic data suggest a deep phylogenetic division between western chimpanzees and those from central and eastern communities, the latter of which do not appear to possess an equivalent phylogenetic division. Given this, Lycett et al. (2010) predicted that if the behavioral data are to be in congruence with the genetic data, phylogenetic structure should be greater when all 10 P. troglodytes communities are examined than when those from just central and eastern regions are analyzed.

Lycett et al. (2010) utilized a combination of the RI statistic, the phylogenetic bootstrap, and the permutation probability test. All three sets of analyses were inconsistent with the prediction of the genetic hypothesis. The MP cladograms returned when all 10 communities were included had an RI of .56, whereas this increased to .68 when only the central and eastern communities were analyzed. This increase is the direct opposite of what is expected under the genetic hypothesis. Likewise, both the phylogenetic bootstrap and the permutation test indicated lower phylogenetic structure when all 10 communities were included than when only the central and eastern communities were analyzed. Hence, both of these randomization procedures were consistent with the RI analysis in producing results in direct opposition to what is predicted by the genetic hypothesis.

In summary, the phylogenetic analyses of chimpanzee behavioral data undertaken to date - in which multiple quantitative measures of phylogenetic signal and alterna- 
tive data sets have been used-have all been consistent in their rejection of the key prediction of the genetic hypothesis. In all three studies, Lycett et al. (2007, 2009, 2010) argued that the mismatch between genetic structure and behavioral patterning was explicable in terms of social learning when the migration pattern of female chimpanzees was taken into account. That is, in contrast to the philopatric pattern of male chimpanzee residence, female chimpanzees typically migrate to another community upon reaching sexual maturity. This contrasting pattern of male versus female residency suggests that female chimpanzees are the primary means by which both genes and culture may feasibly be transmitted intercommunally. Hence, the mismatch is explicable in terms of differing transmission mechanisms for genes and behavioral attributes. As was discussed by Lycett et al. (2007, 2009, 2010), such different mechanisms might involve female chimpanzees abandoning the behaviors of their natal group upon migration, choosing instead to take up behaviors observed in their new peers. Alternatively, or perhaps in addition to this, little attention may be paid to immigrant females, such that any novel behavior patterns exhibited do not spread to their new community. However, at present, our understanding of precisely which of these mechanisms (or combination thereof) are dominant remains limited, and the study of chimpanzee social learning would be advanced by further studies designed to address these issues (Lind \& Lindenfors, 2010; Lycett et al., 2010).

\section{Discussion: The Importance of History in Definitions of Culture}

Phylogenetic studies designed to test a genetic hypothesis for chimpanzee behavioral diversity suggest that genetics is not the dominant causative factor. Indeed, the mismatch between phylogenetic analyses of behavior and genetics is in line with a growing body of experimental evidence from studies of both captive (e.g., Hopper et al., 2007; Horner et al., 2006; Whiten et al., 2007) and wild (e.g., Biro et al., 2003; Biro et al., 2006) animals, suggesting that these behaviors are socially learned. This does not necessarily rule out that in relatively rare cases, specific individual behaviors are influenced by genetics (Laland et al., 2009), but it does argue strongly against genetics as a blanket explanation for wild chimpanzee behavioral patterns. What phylogenetic analyses of chimpanzee behavior support, therefore, is the existence of multiple socially learned traditions that differ intercommunally in terms of exhibition, thus fulfilling some definitions of culture (Whiten \& van Schaik, 2007). Given the controversial nature of defining culture, however, a question remains as to whether it is (scientifically) useful to define these patterns as cultural. A phylogenetic perspective may be informative in this regard.

Whiten and van Schaik (2007) argued that a key feature of some primates is the existence of multiple socially learned behaviors, a distinction that they argued to more meaningfully qualify specific primate species for the label of culture, including chimpanzees. As noted earlier, under strict application of this definition, animals that exhibit only a single — even unambiguous - socially learned tradition in the wild would be excluded as cultural creatures. However, although chimpanzee cultures may differ quantitatively from many other animal traditions, on the basis of this alone it might be difficult to argue that they differ qualitatively in any meaningful sense from any other instance of tradition based on social learning. In the absence of such a distinction, claims that cultures such as those seen in chimpanzees are fundamentally different from other well-documented instances of animal social learning might cynically be open to the claim that some primatologists are merely attempting to pull up the proverbial drawbridge, having seemingly ushered certain primate taxa (i.e., chimpanzees and orangutans) into the cultural castle (despite the fact that Whiten \& van Schaik, 2007, were keen to emphasize that their definition did not necessarily limit culture to the primate order).

Meanwhile, Premack and Premack (1994, p. 350) argued that "History is a sequence of changes through which a species passes while remaining biologically stable." They went on to argue that "one could not write a history of the chimpanzee" (Premack \& Premack, 1994, p. 350). Likewise, Tomasello (1999, p. 512) argued that "human cultures are historical products built up over many generations." Implicitly, therefore, a perceived lack of history excludes chimpanzees from having true culture.

Taking these various factors into account is pertinent when considering phylogenetic analyses of chimpanzee behavioral patterns. Phylogenetics is not merely the organization of units into subsets that represent similarity or difference. Rather, it is a means of organizing evolving units into a hierarchical structure that reflects the genealogy of an evolutionary process (A. B. Smith, 1994). Fundamentally, therefore, phylogenetics is a historical approach to historical questions: how and potentially why things change over time during their evolution (Lipo, O'Brien, Collard, \& Shennan, 2006; O'Brien et al., 2008). In recent years, an increasing number of workers from different fields (anthropology, archaeology, psychology) have come to recognize that human cultural evolution is a process that occurs via the combination of social transmission (i.e., inheritance), variation (e.g., presence in some communities, absence in others), and the differential representation of transmitted elements in subsequent generations (i.e., sorting of variants via natural selection, artificial selection, or drift) (Eerkens \& Lipo, 2007; Lycett, 2009; Mesoudi, Whiten, \& Laland, 2004; Shennan, 2000). In other words, culture evolves via the same general process that Darwin (1859) termed descent with modification to describe biological evolution. Culture in these terms (i.e., evolved mosaics of multiple behaviors that differ intercommunally) can be viewed as an emergent property of the descent with modification process. Fundamentally, this also makes clear the distinction between genuine evolution (i.e., in terms of a system that allows descent with modification through the concurrent properties of variation, inheritance, and sorting) and the nonevolutionary process of change across time, which may be composed of some, but not all, elements of a genuine evolutionary process. 
It is this qualitative distinction that makes animals, such as chimpanzees, with multiple socially learned traditions different from those that display only one or two socially learned traditions: They have a phylogenetic history, behaviors comprising these mosaic patterns will come and go, culture today is not the same as it was in the past, and culture will not be the same in the future as it is right now. It is under this definition of culture, it is contended here, that chimpanzees possess (although not necessarily exclusively) a behavioral signature that merits being termed culture alongside the human case. Taking into account a possible 39 behavioral variants for chimpanzees, each with a possible three character states (i.e., absent, present, customary), there is a staggering possibility of $3^{39}$ unique community profiles $\left(4.05 \times 10^{18}\right)$ existing across time and space. Given that novel behavioral variants in chimpanzees are regularly observed (e.g., Pruetz \& Bertolani, 2007; Sanz et al., 2004), this is probably a conservative estimate of possible diachronic behavioral variation in the Pan lineage. Phylogenetic trees such as those created from the Whiten et al. (1999) data set document just some of the history of these evolving mosaics of variation, but document it nonetheless.

The importance of this point is not to emphasize-nor automatically create - a distinction between chimpanzees and other animals, but to emphasize that the capacity to produce cultural mosaics in this manner (so defined) is phylogenetically homologous in chimpanzees and humans (i.e., was present in the common ancestor of the two lineages). It may ultimately transpire (and indeed, there is evidence to suggest) that animals other than chimpanzees (including nonprimates) might have culture as defined in these more exacting terms, rather than being defined as involving the social transmission of information alone (van Schaik et al., 2003; Whiten \& van Schaik, 2007).

One potential response to the proposition just made, however, is "How does biological change cross-cut and/or interact with cultural evolution under such a definition?" Others might rephrase this question more directly as "Are we to assume that differences between chimpanzee culture and human culture are simply the product of historical happenstance, rather than biologically evolved properties including innate (i.e., nonlearned) cognitive and psychological differences?" The answer, of course, is no. Indeed, the scientific value of such a definition is that it could refocus effort toward understanding what mechanisms are involved in the transmission, generation of variation, and sorting of cultural variants in the chimpanzee versus human case. Tomasello (1994, p. 314) argued that it may be important to ask whether "the behavioral traditions of various animal species are phylogenetically analogous or homologous." Given, however, that a capacity for culture (as defined here) is, most parsimoniously, phylogenetically homologous (i.e., was present in the common ancestor of extant Pan and Homo sapiens), it may be more appropriate to rephrase this as "What autapomorphic (i.e., uniquely derived) characteristics are involved in the generation of human cultural patterns as opposed to chimpanzee cultural patterns?" Such a definition thus allows for cognitive differences across taxa in terms of the different mechanisms involved in the transmission of cultural variants (e.g., stimulus enhancement, emulation, teaching, imitation, language), mechanisms involved in the generation of variation (e.g., an ability to persistently ratchet), and the sorting of variation (e.g., reflexively via evolved cognitive mechanisms or via evolved capacities that enable a greater extent of conscious and more reflective selection of available variants). Ironically, therefore, this definition of culture shares something with Tomasello's (2009) desire to refocus the debate from arguing over definitions to looking at how mechanisms differ in chimpanzees and humans. It may also, however, help to avoid what Laland and Janik (2006) correctly saw as the naturenurture trap of seeing the phenomenon of cultural variation across species as being devoid of a genetic component. Likewise, it also enables discussion of how cultural variants might come to covary with environmental factors (Laland \& Janik, 2006), since ecological environment can also act as a sorting mechanism on cultural variants, so defined (Humle, 2006; Lycett et al., 2009; Möbius, Boesch, Koops, Matsuzawa, \& Humle, 2008).

\section{Conclusions}

Phylogenetic methods, perhaps both methodologically and theoretically, appear to have added to an already noisy and crowded argument. Given the precise nature of what has been argued here to comprise culture (i.e., multiple traditions forming mosaics of behavioral patterns that collectively evolve via a process of descent with modification), instances in which phylogenetic methods might be applied in precisely the same manner as those of chimpanzees in order to study animal social learning may be relatively limited, and other methods (e.g., Franz \& Nunn, 2009; Kendal, Kendal, Hoppitt, \& Laland, 2009; Matthews, 2009; and those discussed in other articles in the present issue) might be more appropriate in alternative circumstances. Phylogenetic techniques are but one of the useful methods of addressing animal social learning. However, if phylogenetically homoplastic instances of culture - as defined in this article-are supported in taxa more distantly related to humans (e.g., in cetaceans or other animals that have not necessarily seen the same longevity and geographical breadth of field study), examining the cognitive, social, and ecological mechanisms involved in the inheritance, variation, and sorting of the different elements making up the cultural pattern will become important, both for their own sake and as a contrasting evolutionary system to that of primates (including humans). Again, phylogenetic methods - as a tool of historical analysis - will have a role to play in such studies.

\section{AUTHOR NOTE}

I am indebted to Mark Collard and Bill McGrew for generously collaborating with me on the question of chimpanzee culture, the products of which have obviously influenced some of the ideas expressed here. I am also grateful to Rachel Kendal and Jamie Tehrani for helpful comments on a previous version of the manuscript, as well as to Tatyana Humle for illuminating conversations. I alone, however, take responsibility for any inaccuracies or omissions. For continued support and aca- 
demic engagement, I am also grateful to Leslie Aiello, Parth Chauhan, John Gowlett, Alex Mesoudi, Chris Norton, Mike O'Brien, and Noreen von Cramon-Taubadel. A special word of thanks is necessary for Rachel Kendal, who organized the original session on which this issue of Learning \& Behavior is based, as well as her coeditors for their invitation to participate. Correspondence concerning this article should be addressed to S. J. Lycett, Department of Anthropology, University of Kent, Canterbury, Kent, CT2 7NR, England (e-mail: s.j.lycett@kent.ac.uk).

\section{REFERENCES}

ARCHIE, J. W. (1989). A randomization test for phylogenetic information in systematic data. Systematic Zoology, 38, 239-252. doi:10.2307/ 2992285

Argue, D., Morwood, M. J., Sutikna, T., Jatmiko, \& SaPtomo, E. W. (2009). Homo floresiensis: A cladistic analysis. Journal of Human Evolution, 57, 623-639. doi:10.1016/j.jhevol.2009.05.002

Biro, D., Inoue-Nakamura, N., Tonooka, R., Yamakoshi, G., Sousa, C., \& MatsuzaWa, T. (2003). Cultural innovation and transmission of tool use in wild chimpanzees: Evidence from field experiments. Animal Cognition, 6, 213-223. doi:10.1007/s10071-003 $-0183-\mathrm{x}$

Biro, D., Sousa, C., \& Matsuzawa, T. (2006). Ontogeny and cultural propagation of tool use by wild chimpanzees at Bossou, Guinea: Case studies in nut cracking and leaf folding. In T. Matsuzawa, M. Tomonaga, \& M. Tanaka (Eds.), Cognitive development in chimpanzees (pp. 476-508). New York: Springer.

BoEsch, C. (1991). Teaching among wild chimpanzees. Animal Behaviour, 41, 530-532. doi:10.1016/S0003-3472(05)80857-7

BoEsCH, C. (1996). The emergence of cultures among wild chimpanzees. Proceedings of the British Academy, 88, 251-268.

Boesch, C., \& Boesch-Achermann, H. (2000). The chimpanzees of the Taï Forest: Behavioural ecology and evolution. Oxford: Oxford University Press.

Boesch, C., Head, J., \& Robbins, M. M. (2009). Complex tool sets for honey extraction among chimpanzees in Loango National Park, Gabon. Journal of Human Evolution, 56, 560-569. doi:10.1016/ j.jhevol.2009.04.001

Bonnie, K. E., Horner, V., Whiten, A., \& de Waal, F. B. M. (2007). Spread of arbitrary conventions among chimpanzees: A controlled experiment. Proceedings of the Royal Society B, 274, 367-372. doi:10.1098/rspb.2006.3733

Brower, A. V. Z. (2000). Homology and the inference of systematic relationships: Some historical and philosophical perspectives. In R. Scotland \& R. T. Pennington (Eds.), Homology and systematics: Coding characters for phylogenetic analysis (pp. 10-21). London: Taylor \& Francis.

Brown, C., \& Laland, K. N. (2003). Social learning in fishes: A review. Fish \& Fisheries, 4, 280-288. doi:10.1046/j.1467-2979.2003 .00122.x

Brumann, C. (2002). On culture and symbols. Current Anthropology, 43, 509-510. doi:10.1086/340282

Call, J., \& Tomasello, M. (1998). Distinguishing intentional from accidental actions in orangutans (Pongo pygmaeus), chimpanzees (Pan troglodytes), and human children (Homo sapiens). Journal of Comparative Psychology, 112, 192-206. doi:10.1037/0735-7036.112.2.192

Corbey, R. (2005). The metaphysics of apes: Negotiating the animalhuman boundary. Cambridge: Cambridge University Press.

DARWIN, C. (1859). On the origin of species. London: John Murray.

DE WAAL, F. B. M. (1999). Cultural primatology comes of age. Nature, 399, 635-636. doi:10.1038/21310

Eerkens, J. W., \& Lipo, C. P. (2007). Cultural transmission theory and the archaeological record: Providing context to understanding variation and temporal changes in material culture. Journal of Archaeological Research, 15, 239-274. doi:10.1007/s10814-007-9013-Z

Faith, D. P., \& Cranston, P. S. (1991). Could a cladogram this short have arisen by chance alone? On permutation tests for cladistic structure. Cladistics, 7, 1-28. doi:10.1111/j.1096-0031.1991.tb00020.x

FARRIS, J. S. (1989a). The retention index and homoplasy excess. Systematic Zoology, 38, 406-407. doi:10.2307/2992406

FARRIS, J. S. (1989b). The retention index and the rescaled consistency index. Cladistics, 5, 417-419. doi:10.1111/j.1096-0031.1989 .tb00573.x
Franz, M., \& NunN, C. L. (2009). Network-based diffusion analysis: A new method for detecting social learning. Proceedings of the Royal Society B, 276, 1829-1836. doi:10.1098/rspb.2008.1824

Gagneux, P., Gonder, M. K., Goldberg, T. L., \& Morin, P. A. (2001) Gene flow in wild chimpanzee populations: What genetic data tell us about chimpanzee movement over space and time. Philosophical Transactions of the Royal Society B, 356, 889-897. doi:10.1098/ rstb.2001.0865

Gagneux, P., Wills, C., Gerloff, U., Tautz, D., Morin, P. A., BoEsch, C., ET AL. (1999). Mitochondrial sequences show diverse evolutionary histories of African hominoids. Proceedings of the $\mathrm{Na}$ tional Academy of Sciences, 96, 5077-5082.

Galef, B. G. (1992). The question of animal culture. Human Nature, 3, 157-178. doi:10.1007/BF02692251

GaLEF, B. G. (2009). Culture in animals? In K. N. Laland \& B. G. Galef (Eds.), The question of animal culture (pp. 222-246). Cambridge, MA: Harvard University Press.

Gilbert, C. C., \& Rossie, J. B. (2007). Congruence of molecules and morphology using a narrow allometric approach. Proceedings of the National Academy of Sciences, 104, 11910-11914. doi:10.1073/ pnas.0702174104

Gonder, M. K., Disotell, T. R., \& OAtes, J. F. (2006). New genetic evidence on the evolution of chimpanzee populations and implications for taxonomy. International Journal of Primatology, 27, 1103-1127. doi:10.1007/s10764-006-9063-y

Goodall, J. (1986). The chimpanzees of Gombe: Patterns of behavior. Cambridge, MA: Harvard University Press.

Hohmann, G., \& Fruth, B. (2003). Culture in bonobos? Betweenspecies and within-species variation in behavior. Current Anthropology, 44, 563-571. doi: 10.1086/377649

Hopper, L. M., Spiteri, A., Lambeth, S. P., Schapiro, S. J., Horner, V., $\&$ Whiten, A. (2007). Experimental studies of traditions and underlying transmission processes in chimpanzees. Animal Behaviour, 73, 1021-1032. doi:10.1016/j.anbehav.2006.07.016

Hoppitt, W. J. E., Brown, G. R., Kendal, R. L., Rendell, L., Thornton, A., Webster, M. M., \& Laland, K. N. (2008). Lessons from animal teaching. Trends in Ecology \& Evolution, 23, 486-493. doi:10.1016/j.tree.2008.05.008

Horner, V., Whiten, A., Flynn, E., \& de WaAl, F. B. M. (2006). Faithful replication of foraging techniques along cultural transmission chains by chimpanzees and children. Proceedings of the National Academy of Sciences, 103, 13878-13883. doi:10.1073/pnas.0606015103

Humle, T. (2006). Ant dipping in chimpanzees: An example of how microecological variables, tool use, and culture reflect the cognitive abilities of chimpanzees. In T. Matsuzawa, M. Tomonaga, \& M. Tanaka (Eds.), Cognitive development in chimpanzees (pp. 452-475). New York: Springer.

Kendal, R. L. (2008). Animal "culture wars." The Psychologist, 21, 312-315

Kendal, R. L., Kendal, J. R., Hoppitt, W., \& Laland K. N. (2009). Identifying social learning in animal populations: A new "option-bias" method. PLOS ONE, 4, e6541. doi:10.1371/journal.pone.0006541

Kenward, B., Weir, A. A. S., Rutz, C., \& Kacelnik, A. (2005). Behavioural ecology: Tool manufacture by naive juvenile crows. Nature, 433, 121. doi: $10.1038 / 433121$ a

Kitching, I. J., Forey, P. L., Humphries, C. J., \& Williams, D. M. (1998). Cladistics: The theory and practice of parsimony analysis (2nd ed.). Oxford: Oxford University Press.

Koops, K., McGrew, W. C., \& MatsuZawa, T. (2010). Do chimpanzees (Pan troglodytes) use cleavers and anvils to fracture Treculia africana fruits? Preliminary data on a new form of percussive technology. Primates, 51, 175-178. doi:10.1007/s10329-009-0178-6

Krützen, M., Mann, J., Heithaus, M. R., Connor, R. C., Bejder, L., \& SHERwIN, W. B. (2005). Cultural transmission of tool use in bottlenose dolphins. Proceedings of the National Academy of Sciences, 102, 8939-8943.

KrÜTZEN, M., van SchaIK, C., \& Whiten, A. (2007). The animal cultures debate: Response to Laland and Janik. Trends in Ecology \& Evolution, 22, 6. doi:10.1016/j.tree.2006.10.011

Laland, K. N. (2008). Animal cultures. Current Biology, 18, R366R370. doi:10.1016/j.cub.2008.02.049

Laland, K. N., \& GaleF, B. G. (EDs.) (2009). The question of animal culture. Cambridge, MA: Harvard University Press. 
Laland, K. N., \& HoppitT, W. (2003). Do animals have culture? Evolutionary Anthropology, 12, 150-159. doi:10.1002/evan.10111

Laland, K. N., \& JANIK, V. M. (2006). The animal cultures debate. Trends in Ecology \& Evolution, 21, 542-547. doi:10.1016/j.tree.2006 .06 .005

Laland, K. N., \& JaniK, V. M. (2007). Response to Krützen et al.: Further problems with the "method of exclusion." Trends in Ecology \& Evolution, 22, 7. doi:10.1016/j.tree.2006.10.010

Laland, K. N., Kendal, J. R., \& Kendal, R. L. (2009). Animal culture: Problems and solutions. In K. N. Laland \& B. G. Galef (Eds.), The question of animal culture (pp. 174-197). Cambridge, MA: Harvard University Press.

Lind, J., \& LindENFors, P. (2010). The number of cultural traits is correlated with female group size but not with male group size in chimpanzee communities. PLOS ONE, 5, e9241. doi:10.1371/journal .pone.0009241

Lipo, C. P., O’Brien, M. J., Collard, M., \& Shennan, S. J. (2006). Cultural phylogenies and explanation: Why historical methods matter. In C. P. Lipo, M. J. O’Brien, M. Collard, \& S. J. Shennan (Eds.), Mapping our ancestors: Phylogenetic approaches in anthropology and prehistory (pp. 3-16). New Brunswick, NJ: Aldine Transaction.

LiU, J., Rubidge, B., \& LI, J. (2010). A new specimen of Biseridens qilianicus indicates its phylogenetic position as the most basal anomodont. Proceedings of the Royal Society B, 277, 285-292. doi:10.1098/ rspb.2009.0883

Lonsdorf, E. V., Eberly, L. E., \& Pusey, A. E. (2004). Sex differences in learning in chimpanzees. Nature, 428, 715-716. doi:10.1038/ 428715a

LyCETT, S. J. (2009). Are Victoria West cores “proto-Levallois"? A phylogenetic assessment. Journal of Human Evolution, 56, 175-191. doi:10.1016/j.jhevol.2008.10.001

Lycett, S. J., Collard, M., \& McGrew, W. C. (2007). Phylogenetic analyses of behavior support existence of culture among wild chimpanzees. Proceedings of the National Academy of Sciences, 104, 17588-17592. doi:10.1073/pnas.0707930104

LycetT, S. J., Collard, M., \& McGrew, W. C. (2009). Cladistic analyses of behavioural variation in wild Pan troglodytes: Exploring the chimpanzee culture hypothesis. Journal of Human Evolution, 57, 337349. doi:10.1016/j.jhevol.2009.05.015

LycetT, S. J., Collard, M., \& McGrew, W. C. (2010). Are behavioral differences among wild chimpanzee communities genetic or cultural? An assessment using tool-use data and phylogenetic methods. American Journal of Physical Anthropology, 142, 461-467. doi:10.1002/ ajpa.21249

MadDEn, J. R. (2008). Do bowerbirds exhibit cultures? Animal Cognition, 11, 1-12. doi:10.1007/s10071-007-0092-5

MatsuZaWa, T. (1994). Field experiments on use of stone tools by chimpanzees in the wild. In R. W. Wrangham, W. C. McGrew, F. B. M. de Waal, \& P. G. Heltne (Eds.), Chimpanzee cultures (pp. 351-370). Cambridge, MA: Harvard University Press.

Matsuzawa, T., Biro, D., Humle, T., Inoue-Nakamura, N., ToNOOKA, R., \& YAMAKoshi, G. (2001). Emergence of culture in wild chimpanzees: Education by master-apprenticeship. In T. Matsuzawa (Ed.), Primate origins of human cognition and behavior (pp. 557574). London: Springer

Matrhews, L. J. (2009). Intragroup behavioral variation in whitefronted capuchin monkeys (Cebus albifrons): Mixed evidence for social learning inferred from new and established analytical methods. Behaviour, 146, 295-324. doi:10.1163/156853909X410937

McGrew, W. C. (1992). Chimpanzee material culture: Implications for human evolution. Cambridge: Cambridge University Press.

McGrew, W. C. (2003). Ten dispatches from the chimpanzee culture wars. In F. B. M. de Waal \& P. L. Tyack (Eds.), Animal social complexity: Intelligence, culture, and individualized societies (pp. 419-439). Cambridge, MA: Harvard University Press.

McGrew, W. C. (2004). The cultured chimpanzee: Reflections on cultural primatology. Cambridge: Cambridge University Press.

McGrew, W. C. (2009). Ten dispatches from the chimpanzee culture wars, plus postscript (revisiting the battlefronts). In K. N. Laland \& B. G. Galef (Eds.), The question of animal culture (pp. 41-69). Cambridge, MA: Harvard University Press.

McLennan, D. A., \& Brooks, D. R. (2001). Phylogenetic systemat- ics: Five steps to enlightenment. In J. M. Adrain, G. D. Edgcombe, \& B. S. Lieberman (Eds.), Fossils, phylogeny, and form: An analytical approach (pp. 7-28). New York: Kluwer Academic.

Mesoudi, A., Whiten, A., \& Laland, K. N. (2004). Is human cultural evolution Darwinian? Evidence reviewed from the perspective of The Origin of Species. Evolution, 58, 1-11. doi:10.1111/j.0014-3820.2004 tb01568.x

Möbius, Y., Boesch, C., Koops, K., Matsuzawa, T., \& Humle, T. (2008). Cultural differences in army ant predation by West African chimpanzees? A comparative study of microecological variables. Animal Behaviour, 76, 37-45. doi:10.1016/j.anbehav.2008.01.008

NishidA, T. (ED.) (1990). The chimpanzees of the Mahale Mountains. Sexual and life history strategies. Tokyo: University of Tokyo Press.

O'Brien, M. J., \& Lyman, R. L. (2003). Cladistics and archaeology. Salt Lake City, Utah: University of Utah Press.

O’Brien, M. J., Lyman, R. L., Collard, M., Holden, C. J., Gray, R. D., \& Shennan, S. J. (2008). Transmission, phylogenetics, and the evolution of cultural diversity. In M. J. O'Brien (Ed.), Cultural transmission and archaeology: Issues and case studies (pp. 39-58). Washington, DC: Society for American Archaeology Press.

Palmer, C. T. (2010). Cultural traditions and the evolutionary advantages of noninnovation. In M. J. O’Brien \& S. J. Shennan (Eds.), Innovation in cultural systems: Contributions from evolutionary anthropology (pp. 161-174). Cambridge, MA: MIT Press.

Premack, D., \& Premack, A. J. (1994). Why animals have neither culture nor history. In T. Ingold (Ed.), Companion encyclopedia of anthropology (pp. 350-365). London: Routledge.

Pruetz, J. D., \& Bertolani, P. (2007). Savanna chimpanzees, Pan troglodytes verus, hunt with tools. Current Biology, 17, 412-417. doi:10.1016/j.cub.2006.12.042

Rendell, L., \& Whitehead, H. (2001). Culture in whales and dolphins. Behavioral \& Brain Sciences, 24, 309-324. doi:10.1017/ S0140525X0100396X

Sanz, C. [M.], Call, J., \& Morgan, D. (2009). Design complexity in termite-fishing tools of chimpanzees (Pan troglodytes). Biology Letters, 5, 293-296. doi:10.1098/rsbl.2008.0786

Sanz, C. M., \& Morgan, D. B. (2007). Chimpanzee tool technology in the Goualougo Triangle, Republic of Congo. Journal of Human Evolution, 52, 420-433. doi:10.1016/j.jhevol.2006.11.001

Sanz, C. [M.], Morgan, D., \& Gulick, S. (2004). New insights into chimpanzees, tools, and termites from the Congo Basin. American Naturalist, 164, 567-581. doi:10.1086/424803

Sargeant, B. L., \& ManN, J. (2009). From social learning to culture: Intrapopulation variation in bottlenose dolphins. In K. N. Laland \& B. G. Galef (Eds.), The question of animal culture (pp. 152-173). Cambridge, MA: Harvard University Press.

Shennan, S. [J.] (2000). Population, culture history, and the dynamics of culture change. Current Anthropology, 41, 811-835. doi:10.1086/ 317403

Smith, A. B. (1994). Systematics and the fossil record: Documenting evolutionary patterns. Oxford: Blackwell Science.

Smith, H. F., \& Grine, F. E. (2008). Cladistic analysis of early Homo crania from Swartkrans and Sterkfontein, South Africa. Journal of Human Evolution, 54, 684-704. doi:10.1016/j.jhevol.2007.10.012

Sperling, E. A., Vinther, J., Moy, V. N., Wheeler, B. M., Sémon, M., Briggs, D. E. G., \& Peterson, K. J. (2009). MicroRNAs resolve an apparent conflict between annelid systematics and their fossil record. Proceedings of the Royal Society B, 276, 4315-4322. doi:10.1098/ rspb.2009.1340

SwOFFord, D. L. (1998). PAUP*: Phylogenetic analysis using parsimony (*and other methods) (Version 4.0) [Computer Software]. Sunderland: Sinauer Associates.

Tebbich, S., Taborsky, M., Fessl, B., \& Blomevist, D. (2001). Do woodpecker finches acquire tool-use by social learning? Proceedings of the Royal Society B, 268, 2189-2193. doi:10.1098/rspb.2001.1738

Tehrani, J. J., \& RiedE, F. (2008). Towards an archaeology of pedagogy: Learning, teaching and the generation of material culture traditions. World Archaeology, 40, 316-331. doi:10.1080/00438240802261267

Terkel, J. (1996). Cultural transmission of feeding behavior in the black rat (Rattus rattus). In C. M. Heyes \& B. G. Galef, Jr. (Eds.), Social learning in animals: The roots of culture (pp. 17-47). San Diego: Academic Press. 
Thornton, A., \& Raihani, N. J. (2010). Identifying teaching in wild animals. Learning \& Behavior, 38, 297-309.

Thouless, C. R., Fanshawe, J. H., \& Bertram, B. C. R. (1989). Egyptian vultures, Neophon percnopterus, and ostrich, Struthio camelus, eggs: The origins of stone throwing behavior. Ibis, 131, 9-15. doi:10.1111/j.1474-919X.1989.tb02737.x

TodD, N. E. (2010). New phylogenetic analysis of the family Elephantidae based on cranial-dental morphology. Anatomical Record, 293, 74-90. doi:10.1002/ar.21010

Tomasello, M. (1994). The question of chimpanzee culture. In R. W. Wrangham, W. C. McGrew, F. B. M. de Waal, \& P. G. Heltne (Eds.), Chimpanzee cultures (pp. 301-317). Cambridge, MA: Harvard University Press

Tomasello, M. (1999). The human adaptation for culture. Annual Review of Anthropology, 28, 509-529. doi:10.1146/annurev.anthro.28.1.509

Tomasello, M. (2009). The question of chimpanzee culture, plus postscript (Chimpanzee culture, 2009). In K. N. Laland \& B. G. Galef (Eds.), The question of animal culture (pp. 198-221). Cambridge, MA: Harvard University Press.

Tutin, C. E. G., Ham, R. M., \& Wrogemann, D. (1995). Tool-use by chimpanzees (Pan t. troglodytes) in the Lopé Reserve, Gabon. Primates, 36, 181-192. doi:10.1007/BF02381344

TuttLe, R. H. (2001). On culture and traditional chimpanzees. Current Anthropology, 42, 407-408. doi:10.1086/320476

TYLOR, E. B. (1871). Primitive culture. London: John Murray.

van Schaik, C. P., Ancrenaz, M., Borgen, G., Galdikas, B., Knott, C. D., Singleton, I., ET AL. (2003). Orangutan cultures and the evolution of material culture. Science, 299, 102-105. doi:10.1126/ science. 1078004

White, J. (2006). Climate: Early and profound human impact? Science, 311, 472. doi:10.1126/science. 1121900

Whiten, A., Goodall, J., McGrew, W. C., Nishida, T., Reynolds, V., Sugiyama, Y., ET AL. (1999). Cultures in chimpanzees. Nature, 399, 682-685. doi: $10.1038 / 21415$

Whiten, A., Goodall, J., McGrew, W. C., Nishida, T., Reynolds, V., SugiYama, Y., ET AL. (2001). Charting cultural variation in chimpanzees. Behaviour, 138, 1481-1516. doi:10.1163/156853901317367717

Whiten, A., Horner, V., \& DE WaAl, F. B. M. (2005). Conformity to cultural norms of tool use in chimpanzees. Nature, 437, 737-740. doi:10.1038/nature04047

Whiten, A., Spiteri, A., Horner, V., Bonnie, K. E., Lambeth, S. P., SchapIRO, S. J., \& DE WAAL, F. B. M. (2007). Transmission of multiple traditions within and between chimpanzee groups. Current Biology, 17, 1038-1043. doi:10.1016/j.cub.2007.05.031

Whiten, A., \& van Schaik, C. P. (2007). The evolution of animal "cultures" and social intelligence. Philosophical Transactions of the Royal Society B, 362, 603-620. doi:10.1098/rstb.2006.1998

Wrangham, R. W., McGrew, W. C., De Waal, F. B. M., \& Heltne, P. G. (EDS.) (1994). Chimpanzee cultures. Cambridge, MA: Harvard University Press.

(Manuscript received January 29, 2010; accepted for publication April 9, 2010.) 